\title{
Efeitos de Dois Parâmetros Antropométricos no Comportamento do Sinal Mecanomiográfico em Testes de Força Muscular
}

\author{
Effects of Two Anthropometric Parameters in the Behavior of the \\ Mechanomyographic Signal on Muscle Force Tests
}

\begin{abstract}
Danielle Polato
Maílson Correa de Carvalho'

Marco Antonio Cavalcanti Garcia'

1. Laboratório de Biomecânica da EEFD/UFRJ

\section{Endereço para correspondência: Marco Antonio Cavalcanti Garcia - Laboratório de Biomecânica da EEFD/UFRJ - Rua Maranhão, 305 \\ - casa 05 - Méier - Rio de Janeiro - RJ - CEP 20720-230 \\ Email: garcia@eefd.ufrj.br marcoacg@unisys.com.br garcia@peb.ufrj.br}

Submetido em 01/12/2006 Versão final recebida em 03/11/2007 Aceito em 17/01/2008

\begin{abstract}
RESUMO
Apesar da mecanomiografia (MMG) ser uma técnica com crescente destaque em investigações relativas ao fenômeno da contração muscular, poucos trabalhos se dedicaram a entender os possíveis efeitos de variáveis antropométricas no sinal de MMG. Portanto, o objetivo do estudo foi avaliar os efeitos da dobra cutânea e do perímetro do braço no comportamento do sinal de MMG nos domínios do tempo e da freqüência em diferentes níveis de contração isométrica. Participaram do estudo 21 indivíduos do sexo masculino (24,9 $\pm 7,8$ anos) e 21 do feminino (20,7 $\pm 2,5$ anos), todos destros. O protocolo experimental constou de um teste de contração voluntária máxima (CVM) que permitiu determinar cinco cargas percentuais administradas durante os testes de força (20\%, 40\%, 60\%, 80\% e 100\% da CVM). Um acelerômetro biaxial foi colocado sobre o ventre muscular do bíceps braquial direito. $\mathrm{O}$ sinal de MMG foi analisado nos domínios do tempo, mediante o comportamento da amplitude do sinal, por meio do cálculo da raiz média quadrática (valor RMS), e da freqüência, através da freqüência mediana (FM) calculada a partir do seu espectro de potência. Estes parâmetros foram extraídos a partir dos sinais gerados na direção X, perpendicular às fibras musculares. Foram também medidos os parâmetros antropométricos dobra cutânea biciptal (BD_DC_B) e perímetros de braço relaxado (BD_PR) e contraído (BD_PC) no terço médio do braço direito. Ambos os grupos apresentaram um comportamento decrescente da FM com o nível de contração. O valor RMS apresentou comportamento crescente com a carga para ambos os grupos. Houve diferenças estatisticamente significativas $(p<0,05)$ entre as variáveis antropométricas quando comparados os grupos. Sugere-se que o valor RMS, ao contrário da FM, que poderá sofrer atenuações por parte dos tecidos presentes na interface entre o músculo e o transdutor, seja mais consistente na discriminação da força muscular.
\end{abstract}

Palavras-chave: mecanomiografia, MMG, sinal de MMG, vibromiografia, VMG.

\begin{abstract}
In spite of being a useful method on muscle contraction analysis, few studies have been dedicated to understand how some anthropometrical parameters can interfere in the mechanomyographic (MMG) signal properties. Therefore, the aim of this study was to evaluate the effects of skinfold thickness and arm circumference in the MMG signal behavior in the temporal and frequency domains at different levels of isometric contraction. Twenty-one males (24.9 \pm 7.8 years) and 21 females (20.7 \pm 2.5 years), all right-handed, participated in this study. Prior to the MMG signals collection, a maximum voluntary contraction test (MVC) was performed to further determine the five levels $(20 \%, 40 \%, 60 \%$, $80 \%$ and $100 \%$ of CVM) used during the tests. The two groups performed isometric contractions in the five different levels while MMG signal was collected from the right biceps brachii muscle by a biaxial accelerometer. The root mean square value (RMS), and the median frequency (MF) calculated from the MMG power spectrum were extracted from the MMG signals in $\mathrm{X}$ direction, which meant perpendicular to the muscle fibres direction. The bicipital skinfold thickness (BD_DC_B), and the circumference of the right arm, with and without contraction of elbow muscles flexors, have also been measured. The MF presented a decreasing behaviour with the increasing level of contraction; the RMS value followed an increasing pattern, though. There were significant statistical differences $(p<0.05)$ for the anthropometric variables between groups. It is suggested that the RMS value, differently from the MF, which can be disrupted by the tissues between the muscle and the transducer, should be better correlated with muscle force level.
\end{abstract}

Keywords: mechanomyography, MMG, MMG signal, vibromyography, VMG. 


\section{INTRODUÇÃO}

Os mecanismos que envolvem o fenômeno da contração muscular ainda constituem um importante objeto de estudo na área da Biomecânica. Mesmo após várias décadas, desde que Huxley e Simmons contribuíram de forma significativa na fundamentação da teoria da contração muscular através do modelo de deslizamento entre miofilamentos ${ }^{(1)}$, ainda é possível encontrar um grande número de trabalhos que visa melhor compreender as propriedades do tecido muscular esquelético na produção de força. Dentre as diferentes técnicas de estudo dos fenômenos que envolvem o processo da contração muscular está a eletromiografia (EMG). A EMG é a técnica que visa detectar e analisar o comportamento da atividade mioelétrica através de sinais colhidos mediante a aplicação de eletrodos sobre o ventre ou no interior do tecido músculo-esquelético ${ }^{(2-4)}$. Logo, submetendo um sujeito a diferentes níveis e tipos de contração, é possível extrair diferentes parâmetros nos domínios do tempo e da freqüência do sinal captado para fins de interpretação dos mecanismos de gradação da força muscular(5,6). Entretanto, outra técnica de análise da contração muscular que vem se popularizando, principalmente nos últimos anos, é a mecanomiografia (MMG).

A MMG se caracteriza pelo uso de transdutores acelerômetros, piezelétricos ou microfones colocados sobre a superfície do músculo ${ }^{(4)}$. Ao contrário da EMG, a MMG visa a análise dos processos reguladores da contração por meio de sinais mecânicos oriundos de vibrações (abalos musculares) laterais e longitudinais geradas pelas fibras musculares, algo que a levou a receber outras denominações, tais como vibromiografia (VMG), aceleromiografia (AMG) e fonomiografia (FMG) (4). Segundo Neering et al. ${ }^{(7)}$, as vibrações têm relação com a redistribuição do material aquoso do sarcoplasma durante o processo de propagação do potencial de ação pela fibra.

Tendo em vista que o sinal de MMG carrega informações que poderão refletir condições tais como força muscular ${ }^{(8)}$, fadiga ${ }^{(9)}$ e dominância ${ }^{(10)}$, diferentes autores têm se dedicado a estudar o potencial desta técnica de forma isolada e/ou complementar à outras técnicas tais como a EMG. Porém, por se tratar de um sinal referente a manifestações de ordem mecânica, as características são bastante diferentes daquelas observadas no sinal de EMG, cujo conteúdo espectral está contido numa faixa entre 25 e $60 \mathrm{~Hz}$, considerada baixa quando comparada àquela apresentada pelo registro eletromiográfico ${ }^{(11)}$.

Com relação ao uso do sinal de EMG na estimativa da produção de força muscular, há um grande número de trabalhos que se dedicam à determinação de parâmetros que sejam consistentes com essa variável| ${ }^{(5,12,13)}$. No entanto há também um grande número de problemas metodológicos no processo de aquisição e processamento do sinal de EMG que o fazem parecer pouco seguro na estimativa da força muscular(6). Da mesma forma que a EMG vem sendo utilizada para este propósito, alguns trabalhos também têm sido conduzidos no uso da MMG como ferramenta na elucidação dos processos envolvidos na gradação da força muscular ${ }^{(8,14)}$. De forma semelhante ao sinal de EMG, o sinal de MMG, por refletir informações de ordem mecânica, poderá estar sujeito a inúmeros efeitos tais como tipagem das fibras musculares ${ }^{(15)}$, comprimento muscular ${ }^{(16)}$ e a outras inúmeras variáveis de ordem antropométrica que até o presente momento foram muito pouco discutidas na literatura ${ }^{(17)}$.

Assim, o objetivo deste trabalho foi avaliar a relação entre o comportamento do sinal de MMG nos domínios do tempo e freqüência, em diferentes níveis de contração isométrica, com dois parâmetros antropométricos, perímetro e dobra cutânea, possivelmente relacionados com a modulação do sinal em ambos os domínios e gêneros.

\section{MATERIAIS E MÉTODOS}

A amostra foi composta por dois grupos, sendo um do sexo masculino ( $\mathrm{n}=21$; idade: $24,9 \pm 7,8$ anos; massa: 72,8 $\pm 11,7 \mathrm{~kg}$; estatura: 1,75 \pm 0,07 m; Índice de Massa Corporal (IMC): 23,82 $\pm 2,82 \mathrm{~kg} \cdot \mathrm{m}^{-2}$ ) e outro do sexo feminino ( $n=21$; idade: de 20,7 \pm 2,5 anos; massa: 61,7 \pm 11,9 kg; estatura: 1,62 \pm 0,04 m; Índice de Massa Corporal (IMC): 23,49 $\left.\pm 3,90 \mathrm{~kg} \cdot \mathrm{m}^{-2}\right)$, alunos do curso de graduação em Educação Física da EEFD/UFRJ, destros, praticantes de atividade física regular e sem histórico de lesões do sistema músculo-esquelético de membros superiores. Por representaram uma população de alunos do curso de graduação em Educação Física daquela instituição e recrutados por meio de convite feito pelos componentes do grupo de pesquisa em sala de aula, a amostragem foi definida como de "conveniência". Todos os voluntários receberam orientação sobre os procedimentos adotados e assinaram termo de consentimento livre e esclarecido, submetido ao Comitê de Ética do HUCFF/UFRJ.

O sistema de aquisição foi composto de um computador (Pentium IV - $1.8 \mathrm{GHz}$ ), um conversor analógico-digital (Spider 8 - HBM, Alemanha) de 16 bits e faixa dinâmica de $\pm 5 \mathrm{~V}$. Para a captação dos sinais de MMG, foi utilizado um sensor de acelerometria (modelo ADXL202E, Analog Devices, EUA), biaxial, com banda passante de $200 \mathrm{~Hz}$ e sensibilidade de $315 \mathrm{mV} / \mathrm{g}$ (g=aceleração da gravidade). O sensor foi montado sobre uma pequena placa acrílica, na qual foi construído o circuito, e cuja massa total é de 1,5 grama (figura 1). Este acelerômetro permite medir acelerações de até duas vezes a aceleração da gravidade (2g). A freqüência de amostragem foi de $600 \mathrm{~Hz}$. Os programas de aquisição e processamento dos sinais, assim como o desenvolvido para a realização dos testes de força, foram elaborados em LabVIEW ${ }^{\text {m }}$ 6i (NATIONAL INSTRUMENTS'", EUA).

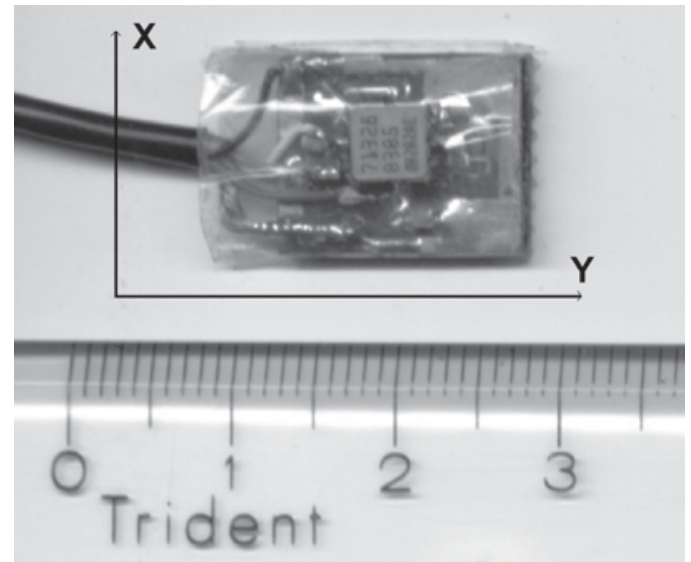

Figura 1. Acelerômetro, cuja massa total é igual a 1,5 grama, e os eixos definidos quanto à capacidade de medição em termos de direções ( $X$ - vibrações laterais/ perpendiculares às fibras musculares). A direção $Y$, correspondente às vibrações longitudinais às fibras musculares, não foi coletada. Uma régua foi colocada ao lado do acelerômetro para uma melhor visualização das suas dimensões.

Para a realização dos testes foi utilizado um aparato mecânico para apoio do membro superior e utilizado um sistema de dinamometria, com capacidade para regulagem individual de altura e distância em relação à articulação do ombro, mantendo-o em flexão de aproximadamente $70^{\circ}$ (figura 2). Um dinamômetro (KRATOS DINAMÔMETROS, Ltda.), fixado ao solo, junto ao suporte, foi utilizado para a aquisição dos sinais de força. O cabo, fixado ao punho do voluntário, era ajustado de forma a permanecer em ângulo de $90^{\circ} \mathrm{com}$ o antebraço. Durante a realização dos testes, cada voluntário permanecia sentado, com as articulações do joelho e quadril flexionadas em aproximadamente $90^{\circ}$; e membro superior flexionado, segundo a figura 2. 


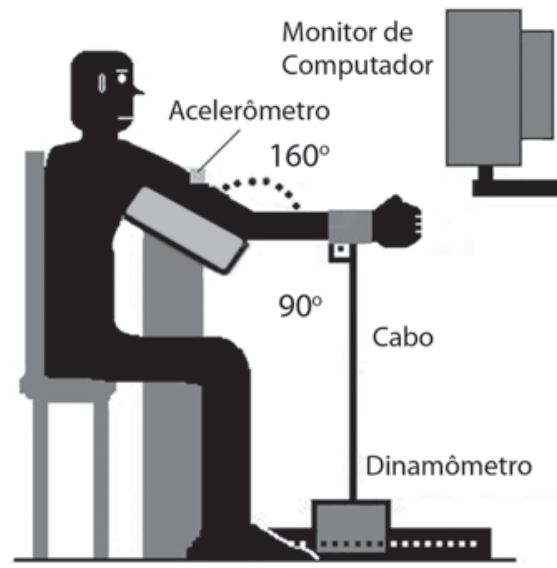

Figura 2. Esquema do aparato mecânico e do posicionamento do voluntário em relação ao sistema de aquisição de força.

O protocolo experimental constou de um teste de contração voluntária máxima (CVM) por 6 segundos com o membro superior dominante. A partir deste dado foram calculadas as cargas percentuais administradas durante os testes de força: 20\%, 40\%, 60\%, 80\% e 100\% da CVM. Os testes foram realizados em um único dia e cada contração, do tipo isométrica, também somente aplicada ao membro superior dominante, foi mantida por 8 segundos, havendo um intervalo mínimo de dois minutos entre cada contração. Para o alcance da carga alvo, cada voluntário recebeu "feedback" visual com o auxílio de um monitor de computador (figura 2), colocado a uma distância de aproximadamente $1 \mathrm{~m}$ do mesmo e ao nível dos olhos, para cada uma das cargas, administradas em ordem aleatória, mediante sorteio realizado antes de cada aquisição.

A aquisição dos sinais de força e de MMG foi definida a partir do alcance da carga alvo, perdurando por 8 segundos. Além disso, todos os voluntários receberam estímulo verbal por parte do experimentador.

Dada a inexistência de um protocolo específico para a colocação deste tipo de transdutor, o mesmo foi colocado sobre a região ventral do músculo bíceps braquial direito por meio de uma fita dupla-face, seguindo o protocolo sugerido por Hermens et al.(6) para a colocação de eletrodos de superfície em aquisição de sinais de EMG, como ressaltado por Matta et al.(14).

Para a análise dos dados, foram somente estudados os 6 segundos intermediários de cada sinal obtido, excluindo, portanto, o primeiro e último segundo. Tal procedimento foi adotado para que se pudesse alcançar a menor variabilidade possível dos sinais de força e de $\mathrm{MMG}^{(14)}$.

Quanto aos parâmetros antropométricos, foram medidos: dobra cutânea (compasso de dobra cutânea CESCORF ${ }^{\circ}$ Ltda. - resolução 0,1 mm) da região sobre o ventre do músculo bíceps braquial direito (BD_DC_B - dobra biciptal direita), referente à marca definida para a colocação do acelerômetro e sendo esta medida realizada com o membro superior (MMSS) em posição anatômica. Também foram tomados os perímetros de braço relaxado (BD_PR) e contraído (BD_PC) a partir de seu terço médio. O perímetro de braço contraído, diferentemente da condição relaxada, foi realizado com as articulações do ombro e cotovelo em flexão de $90^{\circ}$. Em seguida, o próprio voluntário era orientado a realizar resistência manual por meio do segmento contra-lateral e produzir máxima contração da musculatura flexora de cotovelo, sem movimento da mesma. Estes procedimentos foram efetuados sempre pelo mesmo sujeito, sendo este treinado e com experiência prévia em relação aos protocolos.

Foram calculados os seguintes parâmetros a partir dos sinais de MMG: A freqüência mediana (FM), a partir do espectro de potência do sinal, e a raiz média quadrática (valor RMS). Cada um destes parâmetros foi, por sua vez, extraído de janelas de 1 segundo dos trechos intermediários selecionados e a média aritmética de todos os trechos considerada para análise, também como sugerido por Matta et al. ${ }^{(14)}$. Ambos os parâmetros foram extraídos, arbitrariamente, dos sinais de MMG na direção $X$, sendo esta perpendicular à direção das fibras musculares.

A FM foi obtida a partir do espectro de potência do sinal $\left(S_{m}(f)\right)$, calculado via Transformada Rápida de Fourrier (FFT). Esta variável pode ser expressa da seguinte maneira (equação 1):

$$
\int_{0}^{F M} S_{m}(f) d f=\int_{F M}^{\infty} S_{m}(f) d f
$$

Onde, $S_{m}(f)$ é a função de densidade espectral do sinal calculada para cada 1 segundo $(T)$.

O valor RMS, por sua vez, foi obtido com base na seguinte equação (equação 2):

$$
M M G_{\text {RMS }}=\sqrt{\frac{1}{T} \int_{0}^{T} x^{2}(t) d t}
$$

Onde, $x(t)$ é o trecho de sinal no segmento de duração $T$, para $T$ igual a 1 segundo.

A análise dos dados foi realizada por meio do programa STATISTICA "student version" (StatSoft", Inc., EUA). Foram aplicados os seguintes testes estatísticos: Análise de Variância (ANOVA) "two-way" (FM e RMS nos diferentes porcentuais da CVM (\% da CVM) em ambos os grupos) e teste "post-hoc" de Tukey na presença de diferença estatística; os níveis \% da CVM e o gênero foram definidos como fatores na ANOVA; teste T para amostras independentes (Parâmetros Antropométricos e 100\% da CVM $\times$ Grupos; $\mathrm{H}_{0}=$ ausência de diferença entre os parâmetros antropométricos e 100\% da CVM entre os Grupos; Idade, Massa, Estatura e IMC $\times$ Grupos; $\mathrm{H}_{0}=$ ausência de diferença para as variáveis Idade, Massa, Estatura e IMC entre os Grupos); e correlação linear simples (Correlação de Pearson). O nível de significância ( $\alpha$ ) adotado foi de $5 \%$.

\section{RESULTADOS}

A tabela 1 apresenta os valores médios ( \pm Desvios-Padrão) das variáveis FM e RMS nos diferentes níveis \% da CVM.

\begin{tabular}{|c|c|c|c|c|c|c|c|c|c|c|}
\hline Grupo & $\begin{array}{l}\text { RMS 20\% } \\
\left(m . s^{-2}\right)\end{array}$ & $\begin{array}{l}\text { RMS } 40 \% \\
\left(m \cdot s^{-2}\right)\end{array}$ & $\begin{array}{l}\text { RMS 60\% } \\
\left(m \cdot s^{-2}\right)\end{array}$ & $\begin{array}{l}\text { RMS 80\% } \\
\left(m . s^{-2}\right)\end{array}$ & $\begin{array}{c}\text { RMS } 100 \% \\
\left(m . s^{-2}\right)\end{array}$ & $\begin{array}{l}\text { FM 20\% } \\
\text { (Hz) }\end{array}$ & $\begin{array}{l}\text { FM 40\% } \\
\text { (Hz) }\end{array}$ & $\begin{array}{l}\text { FM 60\% } \\
(\mathrm{Hz})\end{array}$ & $\begin{array}{l}\text { FM 80\% } \\
(\mathrm{Hz})\end{array}$ & $\begin{array}{l}\text { FM } 100 \% \\
(\mathrm{~Hz})\end{array}$ \\
\hline $\begin{array}{c}\text { Masculino } \\
(n=21)\end{array}$ & $0,015 \pm 0,009$ & $0,04 \pm 0,02$ & $0,051 \pm 0,02$ & $0,061 \pm 0,033$ & $0,069 \pm 0,03$ & $14,73 \pm 3,84$ & $12,74 \pm 3,29$ & $13,15 \pm 3,36$ & $12,51 \pm 3,91$ & $11,57 \pm 2,82$ \\
\hline $\begin{array}{l}\text { Feminino } \\
\qquad(n=21)\end{array}$ & $0,0077 \pm 0,0032$ & $0,02 \pm 0,0091$ & $0,0297 \pm 0,014$ & $0,043 \pm 0,021$ & $0,055 \pm 0,034$ & $17,19 \pm 4,43$ & $11,70 \pm 4,15$ & $10,55 \pm 2,54$ & $9,01 \pm 2,22$ & $9,85 \pm 3,77$ \\
\hline
\end{tabular}

Tabela 1. Valores médios ( \pm Desvios-Padrão) do valor RMS e da FM extraídos de ambos os grupos. 
Quanto ao comportamento da FM com o nível de contração (Figura 3), o grupo feminino apresentou diferenças estatísticas significativas $\left(F_{(4,100)}=17,628 ; p=0,00000\right)$ entre as cargas, havendo destaque apenas para a diferença entre os níveis 20\% da CVM e todos os demais ( $p<$ 0.000134). Quanto ao grupo masculino, houve diferença estatística significativa para a FM apenas entre os níveis $20 \%$ e $100 \%\left(F_{(4,100)}=\right.$ 2,3291; $p=0,0319)$

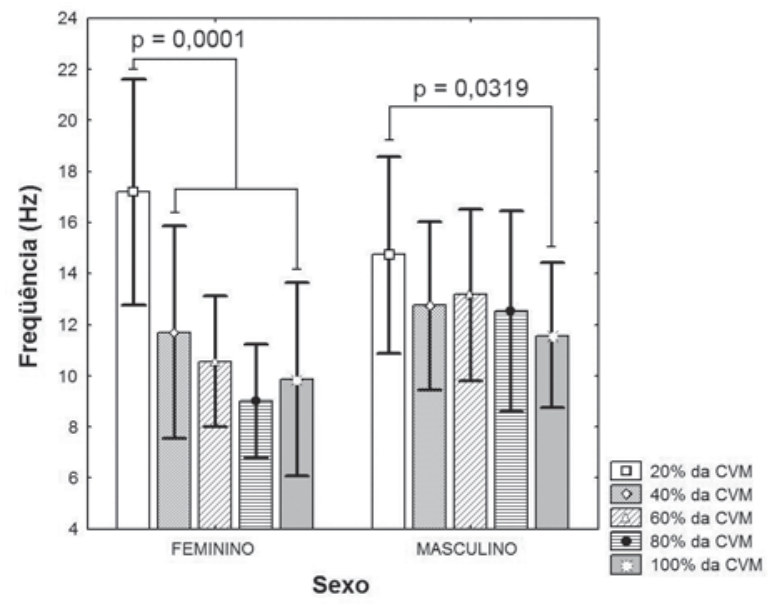

Figura 3. Resultados médios ( \pm desvio padrão) do valor RMS nos cinco diferentes níveis de contração para ambos os sexos.

No que se refere ao valor RMS (figura 4) para o grupo feminino, houve diferenças estatísticas significativas entre os níveis 20\% e 60\% a $100 \%$ da CVM $\left(F_{(4,100)}=18,538 ; p<0,0046\right)$. Quanto ao grupo masculino, houve diferenças estatísticas significativas entre 20\% e 40\% a 100\% da $\operatorname{CVM}\left(\mathrm{F}_{(4,100)}=15,385 ; \mathrm{p}<0,014\right)$.

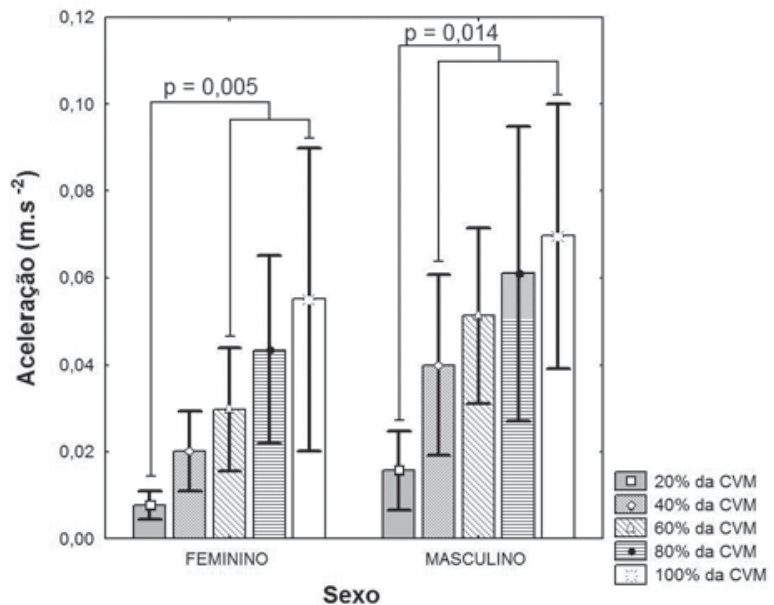

Figura 4. Resultados médios ( \pm desvio padrão) da FM nos cinco diferentes níveis de contração para ambos os sexos.
Os resultados obtidos para ambos os sexos nos domínios da freqüência (figura 3) e do tempo (figura 4) estão de acordo com aqueles apresentados por Matta et al. ${ }^{(14)}$.

Na comparação entre os fatores \% da CVM e gênero para o parâmetro RMS, apesar de haver diferença estatística significativa entre os grupos $\left(F_{(1,200)}=28,093 ; p=0,00000\right)$, ou seja, maiores valores médios para o grupo masculino, não houve interação entre os fatores Gênero e \% da CVM $\left(F_{(4,200)}=0,60344 ; p=0,66059\right)$. Por outro lado, com relação à $F M$, foram observados resultados médios inferiores para o grupo feminino $\left(F_{(1,200)}=7,0262 ; p=0,00868\right)$, além de interação entre os fatores Gênero e \% da CVM $\left(F_{(4,200)}=4,4558 ; p=0,0018\right)$.

A tabela 2, por sua vez, apresenta os valores médios ( \pm DesviosPadrão) das variáveis Idade, Massa, Estatura, IMC, BD_DC_B, BD_PR, BD_PC e 100\% da CVM. Ambos os grupos apresentaram diferenças estatísticas significativas entre as variáveis Idade $(t=2,370 ; p=0,0226)$, Massa ( $t=3,045 ; p=0,0041)$ e Estatura $(t=6,812 ; p=0,000000)$. No entanto, quando comparado o IMC entre os grupos, não houve diferença estatística significativa ( $t=0,314 ; p=0,7549)$.

Quanto às variáveis antropométricas (tabela 2 e figura 5), foram encontradas diferenças estatisticamente significativas entre ambos os grupos para todas as variáveis (BD_PR ( $t=3,468 ; \mathrm{p}=0.001268)$; BD_PC $(t=4,40 ; p=0.000072) ; B D \_D C \_B(t=5,141 ; p=0.000008) ; e 100 \% d a$ CVM ( $t=8,452 ; p=0.000000))$. Destaca-se a diferença para a variável BD_DC_B entre os grupos, que para o grupo feminino foi de 10,17 \pm 4,74 mm e para o masculino foi de 4,66 $\pm 1,26 \mathrm{~mm}$.

Sendo a área de secção transversa de um músculo uma das variáveis que contribui na produção de torque $\operatorname{articular}^{(18)}$ e, portanto, na composição de equações que envolvem o cálculo de força muscular ${ }^{(19)}$, utilizou-se a informação de perímetro braço (BD_PC) para se estimar a relação entre esta e a força máxima (100\% da CVM) produzida e cada

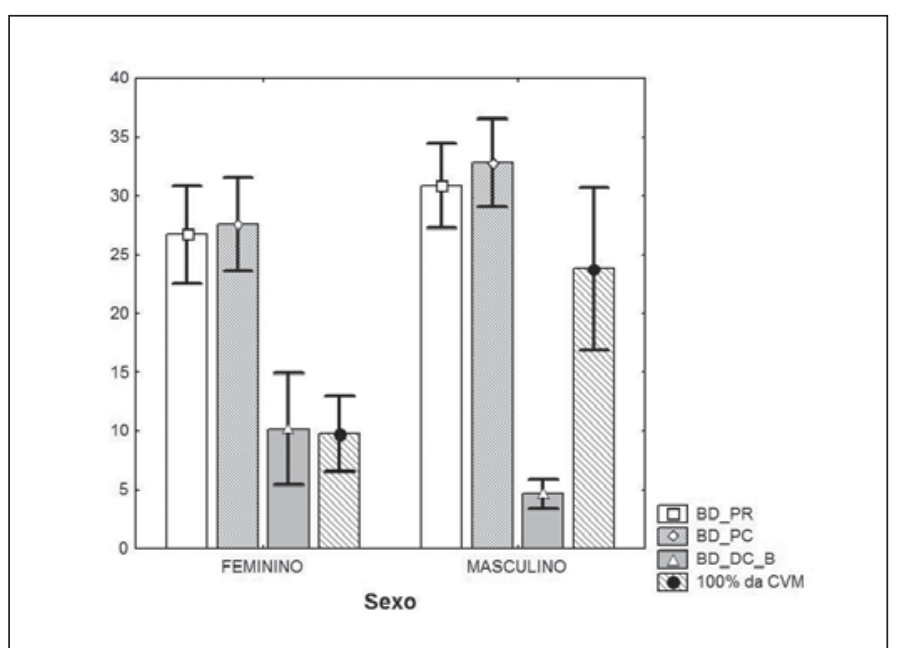

Figura 5. Resultados médios ( \pm desvio padrão) das variáveis perímetro relaxado (BD_PR - unidade: $\mathrm{cm}$ ) e contraído (BD_PC - unidade: $\mathrm{cm}$ ) dos flexores do cotovelo, dobra cutânea biciptal (BD_DC_B - unidade: mm) e contração voluntária máxima (CVM - unidade: kg).

Tabela 2. Valores médios ( \pm Desvios-Padrão) das variáveis antropométricas investigadas e da carga máxima alcançada nos testes de contração voluntária máxima (100\% da (VM) de ambos os grupos.

\begin{tabular}{|c|c|c|c|c|c|c|c|}
\hline Grupo & Idade (anos) & Massa $(\mathrm{kg})$ & Estatura (m) & IMC $\left(\mathrm{kg} \cdot \mathrm{m}^{-2}\right)$ & BD_DC_B $(\mathrm{mm})$ & BD_PC $(\mathrm{cm})$ & $100 \%$ da CVM $(\mathrm{kg})$ \\
\hline $\begin{array}{c}\text { Masculino } \\
(n=21)\end{array}$ & $24,9 \pm 7,78$ & $72,83 \pm 11,73$ & $1,74 \pm 0,073$ & $23,82 \pm 2,82$ & $4,66 \pm 1,25$ & $32,83 \pm 3,75$ & $23,83 \pm 6,91$ \\
\hline $\begin{array}{c}\text { Feminino } \\
(n=21)\end{array}$ & $20,66 \pm 2,55$ & $61,73 \pm 11,88$ & $1,61 \pm 0,042$ & $23,49 \pm 3,9$ & $10,17 \pm 4,47$ & $27,58 \pm 3,98$ & $9,76 \pm 3,22$ \\
\hline
\end{tabular}


um dos parâmetros considerados para análise (FM e RMS em 100\% da CVM). Desta forma, dado que a variável BD_PC apresentou maior correlação positiva ( $r=0.83 ; p=0.0001$ ) com a CVM (em 100\%), foram ajustados polinômios de $1^{\text {a }}$ ordem que permitissem uma melhor visualização do comportamento desta com as variáveis BD_DC_B e FM e o valor RMS em 100\% da CVM (figuras 6 e 7). O nível de 100\% da CVM foi escolhido por atender à condição de máxima contração realizada, justificando o nível de correlação com a variável BD_PC.

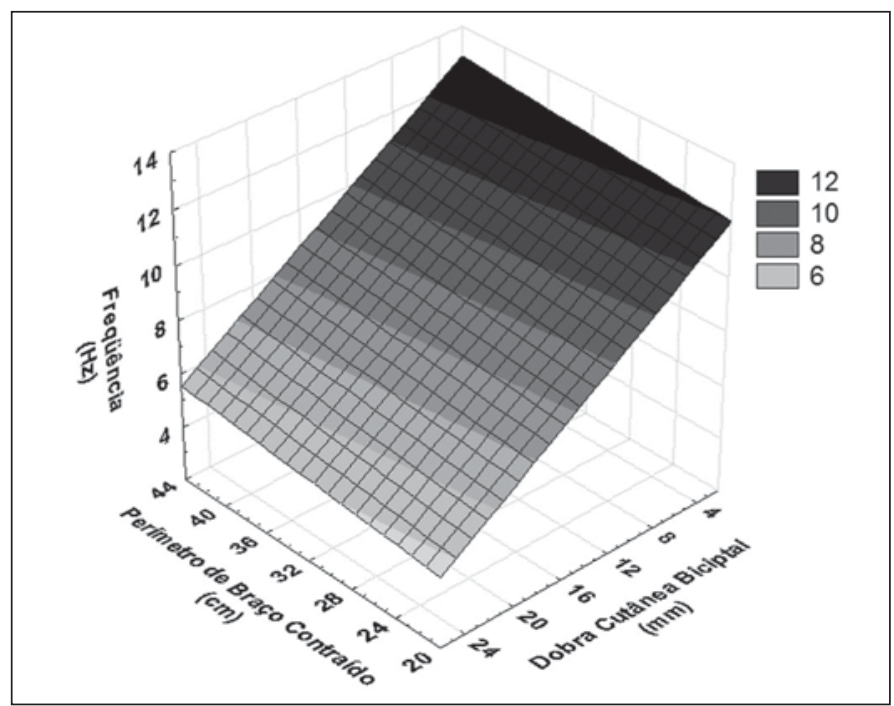

Figura 6. Polinômio de $1^{\mathrm{a}}$ ordem (FM em 100\% da CVM = 11,8098 + 0,0391 BD_PC - 0,3062 BD_DC_B) ajustado às variáveis perímetro contraído (BD_PC - unidade: $\mathrm{Cm}$ ), dobra cutânea biciptal (BD_DC_B - unidade: $\mathrm{mm}$ ) e FM em 100\% da CVM (unidade: $\mathrm{Hz}$ ).

A partir da figura 6 é possível observar uma provável influência da variável dobra cutânea (BD_DC_B) sobre a FM. Nota-se que, para todos os sujeitos com maiores dobras cutâneas, os valores de FM caem consideravelmente $(r=-0,3935 ; p=0,0099)$. O mesmo não acontece com o perímetro de braço $(r=0,0295, p=0,8527)$. Diferentemente da FM, o valor RMS (figura 7) não apontou qualquer tendência clara de parecer sofrer maiores interferências em relação a variável BD_DC_B, onde os sujeitos com maiores perímetros de braço apresentaram maiores valores RMS, sem haver, no entanto, para aqueles sujeitos com maiores e menores dobras diferenças notáveis para seus respectivos valores relativos a este parâmetro.

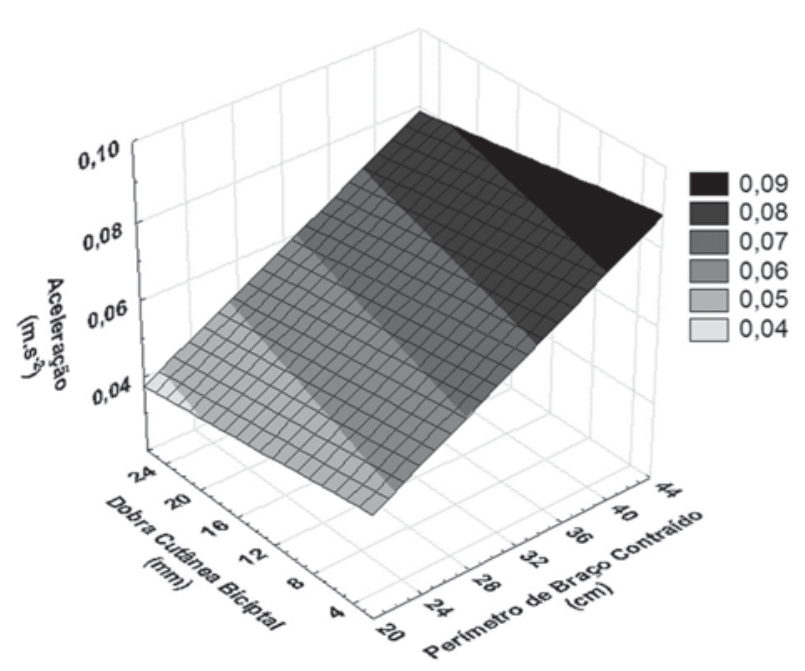

Figura 7. Polinômio de $1^{\mathrm{a}}$ ordem (RMS em 100\% da CVM =0,0134 + 0,0017 BD_PC 0,0004 BD_DC_B ) ajustado às variáveis perímetro contraído (BD_PC - unidade: $\mathrm{cm}$ ), dobra cutânea (BD_DC_B - unidade: mm) e valor RMS em 100\% da CVM (unidade: m.s.-2).

\section{DISCUSSÃO}

O valor RMS, que apresentou comportamento crescente com a carga em ambos os grupos, reflete um aumento na somação e/ou ressonância dos abalos musculares a medida em que mais unidades motoras são recrutadas e quando outras têm um aumento na taxa de disparos de seus potenciais de ação(15). É importante ressaltar que tais abalos seriam em parte produzidos pelo deslocamento do material aquoso presente no sarcoplasma, sendo ele redistribuído à outras partes da fibra muscular. Esta redistribuição conduziria a mudanças no volume de diferentes trechos ao longo de sua estrutura e gerando ondas de pressão, principalmente distribuidas lateralmente e de forma não uniforme ${ }^{(7)}$. Algo que corrobora com esta teoria diz respeito ao comportamento linear e crescente do valor RMS para ambos os grupos. Matta et al. ${ }^{(14)}$ discutem que uma amplitude média superior para sujeitos do sexo masculino seria uma possível prova da presença de uma maior proporção de fibras musculares do tipo II (rápidas) que, por sua vez, contribuiriam não somente para um aumento abrupto do sinal de EMG em maiores níveis de contração quando as unidade motoras rápidas passam a ser recrutadas ${ }^{(12)}$, mas também com o nível de ressonância dos abalos musculares que se refletiriam em um aumento na amplitude do sinal de MMG.

Por outro lado, a FM apresentou um comportamento decrescente e não-linear com o aumento nos níveis de contração. Tal fato vai de encontro à teoria da fusão das fibras que segundo Matta et al. ${ }^{(14)}$, conforme aumenta a taxa de disparos de potenciais de ação das unidades motoras, maior será a somação dos abalos musculares que conduzirão a uma redução em variações abruptas do sinal, sendo este refletido no domínio da freqüência. Entretanto, quando comparados os grupos, tal teoria não é consistente. O grupo feminino apresentou valores médios inferiores àqueles apresentados pelo grupo masculino, exceto em níveis acima de 20\% da CVM. Uma possível hipótese para tal comportamento tem sido dirigida a uma possível diferenciação na composição de fibras musculares do músculo bíceps braquial entre os grupos, como anteriormente discutido. Isto sugeriria a idéia de que as informações contidas no sinal de MMG no domínio da freqüência poderiam refletir a composição de fibras de um determinado músculo(15). No entanto, além de não existir consenso na literatura de que haja uma destacada diferenciação na composição de fibras musculares entre os gêneros e para este músculo, o comportamento da FM em maiores níveis de contração (40 a 100\% da CVM), caso tal diferença existisse, seria diferentes, ou seja, queda mais abrupta para o grupo masculino. Como até o presente momento nenhum trabalho foi conduzido no sentido de comparar diferentes músculos e gêneros por meio do uso de acelerômetros, tal discussão é apenas especulativa.

Não sendo possível estabelecer uma relação entre tipagem de fibras musculares com o comportamento do sinal de MMG nos domínios do tempo e da freqüência, supunha-se inicialmente que ambos os parâmetros extraídos do sinal de MMG pudessem ser sensíveis às variáveis antropométricas analisadas. De forma semelhante aos resultados apresentados por Jaskólska et al.(17), o valor RMS, melhor correlacionado com a força muscular ${ }^{(8,16)}$, parece ser menos susceptível à variável "dobra cutânea", ao contrário da FM. A partir da figura 6 é possível observar uma correlação estatisticamente significativa entre a FM e a dobra cutânea biciptal, mas não com o perímetro de braço, ao contrário do valor RMS, ratificando sua relação com a força muscular (figura 7). Sugere-se que este resultado aponte para uma possível influência deste parâmetro antropométrico na composição espectral do sinal de MMG. Logo, a queda mais abrupta da FM em níveis mais altos de contração para o grupo feminino estaria associada aos maiores 
valores de dobra cutânea que, como discutido, funcionariam como uma forma de filtro, atenuando o comportamento do sinal de MMG no domínio da freqüência.

Sabendo-se que os tecidos adjacentes ao músculo funcionam como um filtro passa-baixas para o sinal de $E M G^{(6)}$, supõe-se que, em se tratando de um sinal de ordem mecânica, o sinal de MMG sofra também dos mesmos efeitos numa intensidade ainda não elucidada. Como o transdutor utilizado neste trabalho foi um acelerômetro, apesar da teoria do processo de fusão mecânica das unidades motoras, onde poderia haver um aumento na freqüência de vibração decorrente do aumento da taxa de disparos dos potenciais de ação quando há um aumento no nível de contração(23), não foi possível confirmar este processo na superfície da pele conforme justificado anteriormente.

Sobre a relação do sinal de EMG na determinação da força muscular, a necessidade de se normalizar os dados e a variabilidade no comportamento das componentes de freqüência e da amplitude com a CVM nos diferentes músculos ainda continua sendo questionada ${ }^{(20,21)}$. Quanto ao sinal de MMG, estas relações ainda não estão totalmente elucidadas e novas alternativas de análise têm sido propostas ${ }^{(22)}$. Como grande parte dos estudos em MMG se baseia na utilização de microfones, estes transdutores parecem não sofrer dos mesmos efeitos relativos à filtragem do sinal originado pelos abalos, o que é ratificado pela literatura que mostra um aumento das componentes de freqüência com o aumento no nível de contração do músculo ${ }^{(15,24)}$, ao contrário de transdutores acelerômetros.

\section{CONCLUSÃO}

Sugere-se que o valor RMS, ao contrário da FM, seja mais consistente na discriminação da força muscular. Mesmo sendo um parâmetro descritor do processo de fusão das fibras musculares, a FM também parece ser sensível à quantidade de tecido adiposo presente na interface transdutor-músculo, cuja quantidade em sujeitos do sexo feminino é maior quando comparada à do sexo masculino. Mesmo assim, discute-se a necessidade de uma avaliação mais detalhada sobre os tecidos presentes nesta interface mediante técnicas tais como o ultra-som, de forma a melhor esclarecer seus reais efeitos no sinal de MMG e melhor avaliar o potencial de aplicação desta técnica nas áreas desportiva e clínica.

Desta forma, conclui-se que, ao se utilizar transdutores acelerômetros na avaliação da força muscular, leve-se em consideração a escolha por parâmetros referentes à amplitude e não no domínio da freqüência, deixando este tipo de abordagem para outros fenômenos relacionados à regulação da contração muscular, tais como dominância, aprendizado e fadiga.

\section{AGRADECIMENTOS}

À divisão de Assistência ao Estudante (DAE) da UFRJ pela concessão das Bolsas de Apoio aos estudantes Maílson Correa de Carvalho e Danielle Polato e à Analog Devices (EUA) por fornecer os acelerômetros.

$\overline{\text { Todos os autores declararam não haver qualquer potencial conflito de }}$ interesses referente a este artigo.

\section{REFERÊNCIAS BIBLIOGRÁFICAS}

1. Huxley AF, Simmons RM. Proposed Mechanism of Force Generation in Striated Muscle. Nature 1971; 233: 533-8.

2. Soderberg GL, Cook TM. Electromyography in Biomechanics. Phys Ther 1984; 64: 1813-20.

3. Webster JG. Encyclopedia of Medical Devices and Instrumentation, $4^{\mathrm{a}}$ Ed. New Jersey: Wiley-Interscience, Inc., 1988.

4. Merletti R, Parker PA. Electromyography: Physiology, Engineering and Noninvasive Applications, New Jersey: John Wiley \& Sons, Inc; 2004.

5. Garcia MAC, Souza MN. Análise do sinal mioelétrico a partir de um parâmetro temporal. Braz J Biomech 2002; 3: 5-12.

6. Hermens HJ, Freriks B, Merletti R, Stegeman D, Blok J, Rau G, et al. European Recommendations for Surface Electromyography - SENIAM Project, 8; 1999.

7. Neering IR, Quesenberry LA, Morris, VA, Taylor SR. Nonuniform volume changes during muscle contraction. Biophy J 1991; 59: 926-32

8. Matheson GO, Maffey-Ward L. Mooney M, Ladly K, Fung T, Zhang YT Vibromyography as a quantitative measure of muscle force production. Scandinavian Journal Rehabil Med 1997; 29:29-35.

9. Orizio C, Gobbo M, Diemont, Esposito F, Vecsteinas A. The surface mechanomyogram as a tool to describe the influence of fatique on biceps brachii motor unit activation strategy. Historical basis and novel evidence. Eur J Physiol 2003: 90:326-36.

10. Garcia MAC, Vargas CD, Souza MN, Imbiriba LA, Oliveira LF. Evaluation of arm dominance by means of the mechanomyographic signal. J Mot Behav 2007, submetido.

11. Oster $\mathrm{G}$, Jaffe JS. Low frequency sounds from sustained contraction of human skeletal muscle. Biophys J 1980; 30: 119-28.

12. Chaffin DB, Lee M, Freivalds A. Muscle strength assessment from EMG analysis. Med Sci Sports Exerc 1980; 12: 205-11.

13. Farina D, Merletti R, Enoka RM. The extraction of neural strategies from surface EMG. J Appl Physio 2004; 96: 1486-95.
14. Matta TT, Perini TA, Oliveira GL, Ornellas JS, Louzada AA, Magalhães J, Imbiriba LA, Garcia MA. Interpretação dos mecanismos de gradação da força muscular através da acelerometria. Rev Bras Med Esporte 2005; 11: 306-10

15. Yoshitake Y, Moritani T. The muscle sound properties of different muscle fiber types during voluntary and electrically induced contractions. J Electromyogr Kinesiol 1999; 9: 209-17.

16. Madeleine P, Bajaj P, Søgaard K, Arendt-Nielsen L. Mechanomyography and electromyography force relationships during concentric, isometric and excentric contraction. J Electromyogr Kinesiol 2000; 10: 33-45

17. Jaskóska A, BrzenczekW, Kisiel-Sajewicz K, Kawczyński A, Marusiak J, Jaskólski A. The Effect of Skinfold on Frequency of Human Muscle Mechanomyogram. J Electromyogr Kinesiol 2004; 14: 217-25.

18. Fukunaga T, Miyatani M, Tachi M, Kouzaki M, Kawakami Y, Kanehisa H. Muscle volume is a major determinant of joint torque in humans. Acta Physiol Scand 2001; 172: 249-55.

19. Mijatovic D, Bulic K, Nikolic V. Quantification model for muscular forces and momentums in human lower extremities. Coll Antropol 2006; 30: 593-9.

20. Erdemir A, McLean S, Herzog W, van den Bogert AJ. Model-based estimation of muscle forces exerted during movements. Clin Biomech 2006; in press.

21. Wakeling JM, Uehli K, Rozitis Al. Muscle fibre recruitment can respond to the mechanics of the muscle contraction. J R Soc Interface 2006; 3: 533-44.

22. Jotta B, Carvalho MC, Polato D, Garcia MAC, Souza MN. Orientação global da aceleração na análise mecanomiográfica da contração muscular. In: Mühlen SS, editor. Proceedings of XX Congresso Brasileiro de Engenharia Biomédica; 2006:1443-6.

23. Yoshitake $Y$, Shinohara M, Eu H, Moritani T. Characteristics of surface mechanomyogram are dependent on development of fusion motor units in humans. J Appl Physiol 2002; 93: 1744-52.

24. Akataki K, Mita K, Watakabe M, Itoh K. Mechanomyogram and force relationship during isometric ramp contractions of the biceps brachii muscle. Eur J Appl Physiol 2001; 84: 19-25. 